\title{
OPEN Regulatory role of the intestinal microbiota in the immune response against Giardia
}

\author{
B. Maertens ${ }^{1}$, A. Gagnaire ${ }^{1}$, O. Paerewijck ${ }^{1}$, K. De Bosscher ${ }^{2}$ \& P. Geldhof ${ }^{1 凶}$
}

Giardia duodenalis is one of the most commonly found intestinal parasites in mammalian hosts. Infections can generally be cleared by mounting an adequate protective immune response that is orchestrated through IL-17A. This study was aimed to investigate if and how the intestinal microbiome affects the protective Th17 response against Giardia by analysing and comparing the immune response following a $G$. muris and $G$. duodenalis infection in antibiotic treated and untreated mice. Depletion of the intestinal flora by antibiotic treatment had a severe effect on the infection dynamics of both Giardia species. Not only duration of infection was affected, but also the parasite burden increased significantly. Markers associated with a protective immune response, such as IL-17A and mannose binding lectin 2 were still significantly upregulated following infection in the antibiotictreated mice, despite the lack of protection. On the other hand, the antibiotic treatment significantly decreased the level of IgA in the intestinal lumen by affecting its transporter and by reducing the number of IgA $\mathrm{A}^{+} \mathrm{B}$-cells at the Peyer's patches. Furthermore, the depletion of the gut microbiota by antibiotics also significantly lowered the intestinal motility. The combination of these factors likely results in a decreased clearance of the parasite from the intestinal tract.

With more than 200 million cases a year worldwide, Giardia duodenalis is one of the most common intestinal parasites in humans, especially in developing countries ${ }^{1}$. Giardia infects both children as adults and causes the enteric infection giardiasis, in which fatty diarrhea, nausea and flatulence are common symptoms ${ }^{2}$. However, many infected individuals do not show any symptoms and function as carriers, suggesting that the true prevalence of Giardia infections is even higher than estimated ${ }^{3}$. Infections occur through the fecal-oral route by ingestion of contaminated food or drinking water. Recent studies have highlighted the importance of interleukin 17A (IL-17A) as the key factor in the development of intestinal immunity against Giardia ${ }^{4,5}$. Both G. muris and G. duodenalis infection studies in mice showed a significant upregulation of IL-17A starting one week after infection. Furthermore, it was shown that mice deficient in the IL-17A receptor or IL-17 itself lose their ability to clear the infection ${ }^{4,5}$. The most important downstream effectors of the IL-17A response, that have been shown to play a critical role in the clearance of a Giardia infection, are the activation of the complement system by mannose-binding lectin 2 (MBL2) and the production and secretion of parasite-specific IgA's ${ }^{4,6,7}$. CD4 + T cells are one of the important cellular sources of IL-17A following a Giardia infection ${ }^{4}$. The differentiation of these Th17 cells is driven by IL- 6 and the retinoic acid-related orphan receptor RORgT, which are both upregulated in an early phase of a Giardia infection ${ }^{5,8}$.

In steady state, Th17 cells are typically abundant in the lamina propria of the small intestine ${ }^{9}$. However the amount of Th17 cells in the lamina propria are extremely reduced in germfree mice and mice treated for four weeks with an antibiotic cocktail of neomycin, ampicillin, vancomycin and metronidazole, indicating that the intestinal microbiome is important for the differentiation of Th17 cells in the small intestine ${ }^{10}$. Furthermore, in mammalian neonates, it has been shown that the microbial colonization of the gut is necessary for the development and education of a functioning immune system ${ }^{11,12}$. These observations suggest that the host intestinal microbiome might also play an important role in the induction of the protective IL-17A response following a Giardia infection. Neonatal mice for example, infected with G. muris, display a prolonged course of infection and a delayed IL-17A response compared to older mice ${ }^{13}$. Furthermore, the oral administration of antibiotics makes mice more susceptible to a $G$. duodenalis infection and results in a higher parasite burden compared to untreated mice ${ }^{14}$.

${ }^{1}$ Department of Virology, Parasitology and Immunology, Laboratory of Parasitology, Faculty of Veterinary Medicine, Ghent University, Merelbeke, Belgium. ${ }^{2}$ VIB Department of Medical Protein Research, Translational Nuclear Receptor Research Lab, Faculty of Medicine and Health Sciences, Ghent University, Ghent, Belgium. ${ }^{\varpi}$ email: peter.geldhof@ugent.be 
In order to gather more insights in the role of the microbiome to mount an anti-Giardia immunity, we analysed and compared the intestinal immune responses following a G. muris infection in antibiotic treated versus untreated mice and we set out to unravel the mechanism by which antibiotic-treated mice are more susceptible to a $G$. duodenalis infection.

\section{Material and methods}

Ethical statement. All animal experiments were conducted in accordance with the European Union (E.U.) Animal Welfare Directives, the VICH Guidelines for good Clinical Practice and in compliance with the ARRIVE guidelines (https://arriveguidelines.org). Ethical approval to conduct the studies was obtained from the Ethical committee of the Faculty of Veterinary Medicine, Ghent University (EC2019/10).

Murine infection studies. All infection studies were performed in female C75 BL/6 mice (Charles River) of 6 weeks old at the time of infection. Before the start of the study, mice were co-housed in groups of 5 animals. Following antibiotic treatment and/or infection, mice were transferred to smaller cages and co-housed with 2 or 3 animals. The antibiotic treatment was administered through the drinking water and consisted of $1.4 \mathrm{mg} / \mathrm{ml}$ neomycin, $1 \mathrm{mg} / \mathrm{ml}$ ampicillin or $1 \mathrm{mg} / \mathrm{ml}$ vancomycin or a combination of all three. Treatment was started five days prior to infection and was kept until the end of the experiment. Mice were infected with either $10^{3} \mathrm{G}$. muris cysts suspended in $0.1 \mathrm{ml}$ phosphate-buffered saline (PBS) or 1 million G. duodenalis (assemblage B-GS/M strain) tropohozoites both by oral gavage. In order to monitor the course of the infection, faecal cyst counts were performed at regular time points (indicated in the Figure legends) following infection. Cysts were isolated from faecal pellets by a centrifugation step at low speed over a $1 \mathrm{M}$ sucrose gradient and microscopically counted with a hemacytometer as previously described by Roberts-Thomson and Mitchell ${ }^{15}$. Animals were euthanized at different timepoints following infection (day 7, 14 and 21) by cervical dislocation. The fifth $\mathrm{cm}$ of the small intestine was isolated and snap-frozen in liquid nitrogen and stored at $-80^{\circ} \mathrm{C}$ for further analysis. The remainder of the small intestine was opened longitudinally and incubated in ice-cold PBS for $20 \mathrm{~min}$ in order to count the number of Giardia trophozoites. Subsequently, when necessary the solution was diluted, followed by counting the number of trophozoites with the use of a hemacytometer.

Bacterial load. 16S rRNA levels were determined in both feces and small intestinal content. For feces, samples were collect at all timepoints, i.e. before the start of the antibiotic treatment (day - 5), at the day of infection (day 0 ) and at day 14 and 21 post G. muris infection. Intestinal content samples were collected at necropsy at day 21 post $G$. muris infection. Both samples were immediately snap-frozen and stored at $-80^{\circ}$. DNA extraction was performed on $0.025 \mathrm{~g}$ feces or $300 \mu \mathrm{l}$ intestinal content using the QIAamp DNA stool mini kit (Qiagen) according to manufacturer's instruction with the addition of a $5 \mathrm{~min}$ heating step at $95^{\circ} \mathrm{C}$ during homogenisation. The quantitative PCR was performed using the StepOnePlus real-time PCR system (Applied Biosciences) under the following conditions: $95^{\circ} \mathrm{C}$ for $10 \mathrm{~min} ; 40$ cycles with 1 cycle of denaturation at $95{ }^{\circ} \mathrm{C}$ for $30 \mathrm{~s}$ and 1 cycle for both annealing and extension at $60^{\circ} \mathrm{C}$ for $1 \mathrm{~min}$.

RNA isolation and qRT-PCR. For intestinal gene expression analysis, a $1 \mathrm{~cm}$ long fragment of the duodenum was taken at $4 \mathrm{~cm}$ from the gastroduodenal junction and snap-frozen in liquid nitrogen. The samples were next disrupted and homogenised using a Tissuelyser II (Qiagen). RNA extraction was subsequently performed using the RNeasy minikit (Qiagen) according to the manufacturer's instructions. Removal of genomic DNA was done by an on-column RNase-free DNase set (Qiagen). Total RNA concentrations were measured using a Nanodrop ND-1000 spectrophotometer (Nanodrop Technologies) and the quality was verified by an Experion automated electrophoresis system (Bio-Rad). To obtain cDNA for qRT-PCR analysis, the iScript cDNA synthesis kit was used (Biorad).

All qRT-PCR analyses were performed using the StepOnePlus real-time PCR system (Applied Biosciences) under the following conditions: $95^{\circ} \mathrm{C}$ for $20 \mathrm{~s} ; 35$ cycles with 1 cycle of denaturation at $95^{\circ} \mathrm{C}$ for $5 \mathrm{~s}$ and 1 cycle for both annealing and extension at $60^{\circ} \mathrm{C}$ (the optimal annealing temperature for all the genes examined). For all reactions, SYBR green master mix (Applied Biosystems) was used with $10 \mathrm{ng}$ of single-stranded cDNA and $500 \mathrm{nM}$ of primer. Primer sets for every gene were designed using Primer3 software in a exon-exon spanning manner in order to control for potential genomic DNA contamination (see Appendix for sequences). All samples were analysed in duplo and a non-template control was included in each assay. Melting curve analyses were performed post-analysis to ensure the specificity of the primers. Relative quantities were calculated out of the

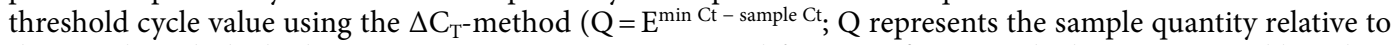
the sample with the highest transcription; E represents amplification efficiency, which was measured based on a standard dilution curve obtained by serial dilutions of pooled cDNA material from all samples; min Ct equals the lowest $C_{T}$ value). The obtained values were normalized in gNORm with the housekeeping genes TATA box binding protein (TBP) and Hypoxanthine phosphoribosyltransferase 1 (HPRT1) as previously described ${ }^{5}$.

Flow cytometry. Peyer's patches (PP) were isolated from the small intestine and cells were extracted as previously described ${ }^{16}$. Briefly, PP were finely cut and incubated for $40 \mathrm{~min}$ at room temperature with collagenase/ DNase. Cells were then filtered through a $70 \mu \mathrm{m}$ cell strainer and pelleted by centrifuging at $300 \times g, 4{ }^{\circ} \mathrm{C}$ during $5 \mathrm{~min}$ and then resuspended in FACS buffer (PBS, 2\% FCS, $5 \mathrm{mM}$ EDTA) and counted before antibody staining. For flow cytometry, cells were first incubated on ice in FACS buffer containing an anti-CD16/CD32 antibody to block the Fc receptor for $10 \mathrm{~min}$ and then incubated $30 \mathrm{~min}$ on ice in the dark with antibodies against the following surface markers : CD45-PE-eF610 (eBiosciences, clone 30-F11), CD19-PerCp-Cy5.5 (Biolegend, clone 6D5), CD3-PeCy7 (eBiosciences, clone 145-2C11), B220-AF647 (BD Biosciences, clone RA3-6B2), IgD-BV421 
(BD Biosciences, clone 11-26c.2a), IgM-FITC (BD Bioscience, clone II/41) and IgA-PE (eBiosciences, clone mA-6E1). Cell viability was evaluated using Fixable Viability Dye eFluor 506 (eBiosciences). Cell acquisition was performed using a CytoFlex (Beckman Coulter) and data were analyzed with the CytoExpert software (Beckman Coulter).

ELISA. Luminal IgA levels from the small intestine were measured by an enzyme-linked immunosorbent assay (ELISA). The small intestine was removed from mice after necropsy and incubated overnight at $4{ }^{\circ} \mathrm{C}$ in $\mathrm{PBS}$ with $0.01 \%$ sodium azide and $1 \%$ protease inhibitor cocktail (Sigma-Aldrich). The IgA containing supernatants were transferred to a new tube after centrifugation at $16,000 \times \mathrm{g}$ for $10 \mathrm{~min}$ and stored at $-80^{\circ} \mathrm{C}$. For the sandwich ELISA, maxisorb 96-well plates were coated with $2 \mu \mathrm{g} / \mathrm{ml}$ sheep anti-mouse IgA (Sigma-aldrich) for $16 \mathrm{~h}$ at $4{ }^{\circ} \mathrm{C}$. After washing with PBS-Tween 20 (0.05\%) (PBST), plates were subsequently blocked with $2 \%$ bovine serum albumin (BSA) in PBST. Luminal extracts were added to the plates in a 1/10 dilution and incubated for $1 \mathrm{~h}$ at room temperature followed by a washing step with PBST. Detection of IgA was performed by adding goat anti-mouse IgA-HRP (Sigma-Aldrich) in a 1/1000 dilution in PBST followed by a visualisation by ABTS in ABTS buffer (Roche). Finally, optical density was measured at $405 \mathrm{~nm}$ with a Tecan plate reader moderated by conjugate control levels measured at $492 \mathrm{~nm}$.

Intestinal motility. To measure the intestinal motility, all mice were starved overnight with water available freely. Mice were orally gavaged with $0.2 \mathrm{ml}$ of $10 \%$ charcoal (Sigma-Aldrich) in $5 \%$ gum acacia (Sigma-Aldrich) and were sacrificed $20 \mathrm{~min}$ after administration. Subsequently, the small intestine was removed and measured from pylorus till the cecum. Next, the distance travelled by the dye was measured from pylorus till the leading edge of the charcoal dye. Motility was set as the percentage of the distance travelled by the dye.

Statistics. Statistical analysis for gene expression, IgA ELISA and flow cytometry were performed by GraphPad Prism software using non-parametric Kruskal-Wallis test followed by a Dunn's multiple-comparison test to determine significant differences between both infected and non-infected mice as between antibiotic treated and non-treated groups. Differences were considered significant at a $p$-value of $\leq 0.05$. For trophozoite counts, area under the curve analysis and intestinal motility a Mann-Whitney $U$ test was performed in order to detect significant differences. A p-value $\leq 0.05$ was considered significant.

\section{Results and discussion}

Reduction of the intestinal microbiota enables $G$. duodenalis infection in mice and prolongs the course of a G. muris infection. The G. duodenalis infection model in adult mice was originally developed to overcome several limitations with existing infection models like G. muris in mice and G. duodenalis in gerbils ${ }^{17}$. Yet also this model encounters limitations as infection by oral ingestion of cysts requires a high amounts of cysts which are difficult ot produce. For this reason, it is typically replaced by oral gavage of trophozoites which can be grown in vitro ${ }^{18}$. Furthermore, it has been described that $G$. duodenalis infection in mice displays a variable success rate, which could be overcome by oral administration of antibiotics, indicating the importance of the intestinal microbiome for susceptibility. Singer et al. ${ }^{14}$ showed that mice with a conventional microbiome from one supplier were susceptible to infection whereas mice from another supplier were not. Later work showed that a WB strain of $G$. duodenalis required an antibiotic treatment prior to infection whereas the GS strain did not ${ }^{15}$.

In this study a $G$. duodenalis infection in C57BL/6 mice with both a conventional microbiome and an antibiotic-depleted microbiome was performed. The results confirmed previously described findings by Singer et al. ${ }^{14}$, that mice with a conventional microbiome indeed did not develop a measurable infection (Fig. 1A) as both parameters indicative of a Giardia infection, namely faecal cysts secretion and trophozoites in the duodenum, were absent. However, antibiotic treatment resulted in a marked infection with cyst secretion in the faeces starting around day 5 post infection (p.i.) and with a peak between day 7 and 10 p.i. followed by a decrease towards day 21 p.i. (Fig. 1A). A similar pattern was also seen for the trophozoite counts with a peak early in the infection and a decrease of the parasite burden at day 21 p.i. (Fig. 1B). These findings indicate an important role for the microbiome in the establishment of a G. duodenalis infection whereas the ability of the animals to clear the infection remains intact.

In a next phase, the same approach was used to investigate the effect of an antibiotic treatment on a G. muris infection, with the inclusion of an additional time point at day 14 p.i. for trophzoites counts in order to compare with previously performed studies ${ }^{5,7}$. Cyst counts (Fig. 1C) of mice without antibiotics displayed a normal pattern with a peak of infection at day 7 p.i., after which the number of cysts in the faeces decreased to the detection limit at day 21p.i. Mice with antibiotic treatment however, showed a delayed peak of cyst secretion at day 13 p.i. followed by a slower decrease of cyst levels. At day 21 p.i., antibiotic treated mice still secreted higher numbers of cysts in their faeces in comparison to untreated mice. Overall, the area under the curve analysis (Fig. 1D) of the cyst counts showed a significant increase in cyst excretion. To monitor the course of infection more closely, trophozoites counts (Fig. 1E) were performed after necropsy at day 7, 14 and 21 p.i. for both groups. A first observation during necropsy was the extreme enlargement of the cecum in all antibiotic treated mice compared to mice with a normal microbiota (Supplementary Data, S1), which has already been described in the past ${ }^{19}$. The number of trophozoites was similar for both groups at day 7 p.i., indicating the onset of infection was not affected by the antibiotic treatment. The untreated mice subsequently showed a steady decrease in the number of trophozoites after day 7 p.i. whereas this was not the case for the antibiotic treated mice. Furthermore, trophozoite counts were both on day 14 and 21 p.i. significantly higher in the antibiotic treated mice compared to the untreated mice. To examine the effect of the antibiotics separately, additional infection studies were performed in which 
A

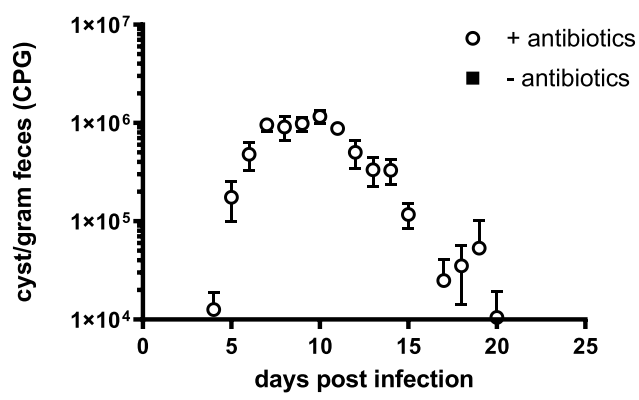

B

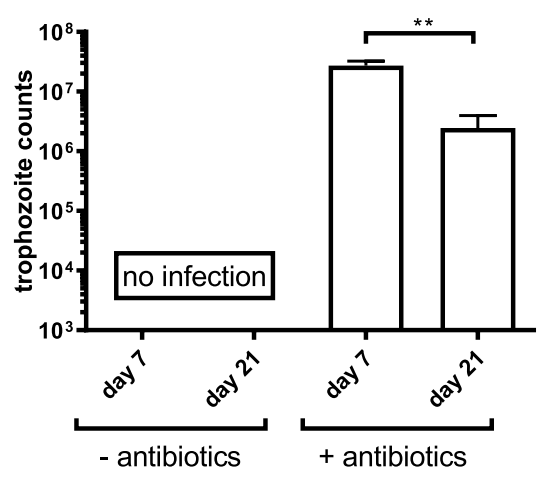

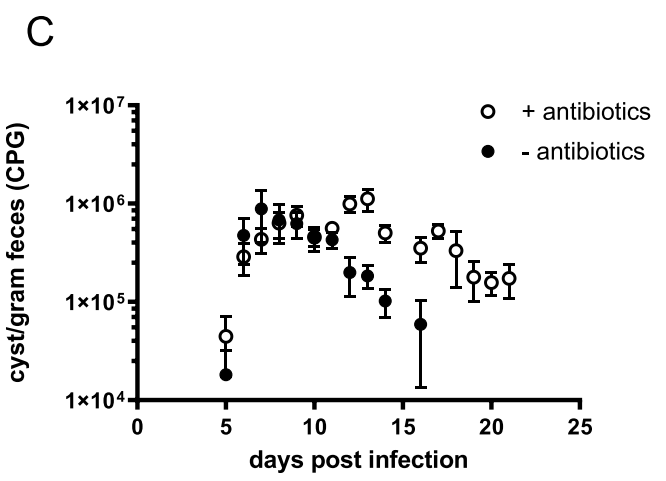
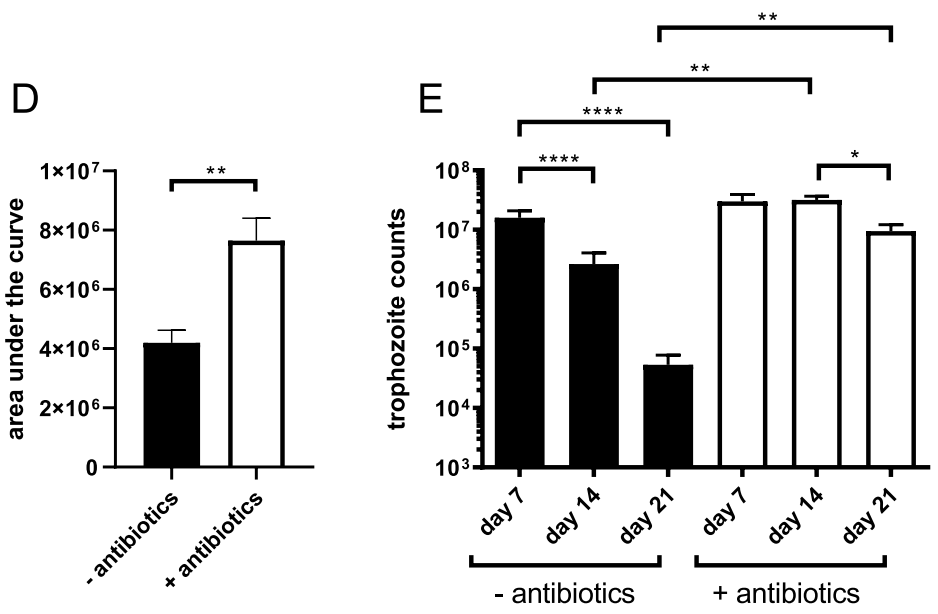

Figure 1. Dynamics of Giardia infections in mice following antibiotic treatment. (A) G. duodenalis cysts present in the feces of antibiotic-treated and untreated mice were monitored daily until day 21 p.i. Mean numbers of cysts per gram faeces obtained from 5 mice at every time point are depicted, with SEM as error bars. (B) G. duodenalis trophozoite numbers in the small intestine of antibiotic-treated C57Bl/6 mice at indicated time points. No trophozoites were detected in the untreated mice. (C) G. muris cysts present in the faeces of antibiotic-treated and untreated mice were monitored from day 4 p.i. until day 21 p.i. Mean numbers of cysts per gram faeces obtained from 5 mice at every time point are depicted, with SEM as error bars. (D) Area under the curve analysis of cyst counts between antibiotic treated and untreated C57Bl/6 mice infected with G. muris (E) G. muris trophozoite numbers in the small intestine of antibiotic-treated and untreated C57Bl/6 mice at indicated time points. $\left({ }^{*} \mathrm{p} \leq 0.05,{ }^{* *} \mathrm{p} \leq 0.01,{ }^{* *} \mathrm{p} \leq 0.001,{ }^{* * *} \mathrm{p} \leq 0.0001\right)$.

neomycin, ampicillin and vancomycin were tested individually. None of the individual antibiotics had an effect on the level of trophozoites present in the small intestine (Supplementary Data, S2). These findings indicate the necessity for the complete cocktail of antibiotics to alter the course of a G. muris infection.

We subsequently wished to determine the effectiveness of the antibiotic treatment by analysing both the total bacterial load as well as some of the more prevalent bacterial phyla typically present in mice (Bacteroidetes, Firmicutes and Beta proteobacteria) by $16 \mathrm{~S}$ rRNA qPCR. As shown in Fig. 2, the antibiotic treatment significantly reduced the total bacterial load present in the feces throughout the whole study (Fig. 2A). A similar effect was also measurable for the Bacteriodetes, Firmicutes and Beta proteobacteria (Fig. 2B-D). In the small intestine, the bacterial load was also strongly reduced, at least at the level of the total bacterial load and the phylum Bacteroides. The decrease for the Firmicutes and Beta proteobacteria on the other hand was smaller. Overall, these results show that the antibiotic treatment resulted in a strong reduction of the bacterial load in the digestive tract, yet the treated mice cannot be considered as 'germfree'.

The gut microbiome triggers the intestinal expression of antimicrobial peptides. Multiple studies have shown in the past that the presence of an intact intestinal microbiome contributes to an adequate innate immune response in the gut ${ }^{20}$. The interaction between the innate immune response and the intestinal microbiome is not only important for the homeostasis of the intestinal environment but also prevents intestinal colonisation of potential pathogenic infections. Therefore, an antibiotic-mediated depletion of the intestinal microbiome could potentially lead to an impaired innate immune barrier and subsequently enable the establishment of for example $G$. duodenalis in mice.

One of the factors of the innate immune system that is important in the defense against a Giardia infection is the production of antimicrobial peptides (AMPs). These small peptides are mainly produced by Paneth cells and 
A

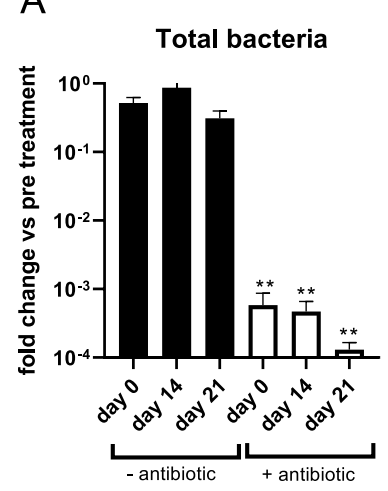

B

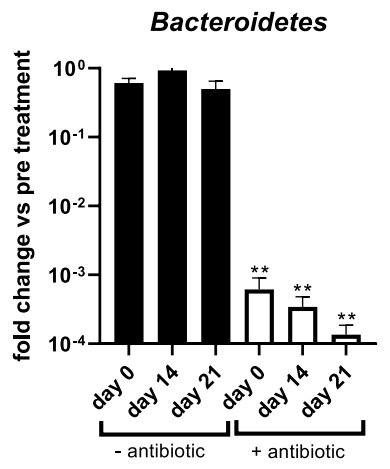

C

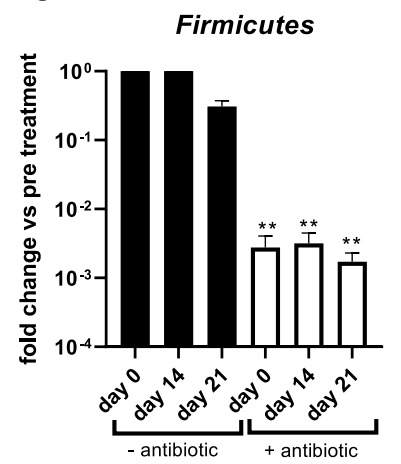

Beta proteobacteria

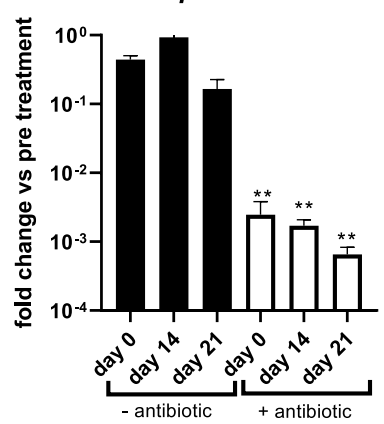

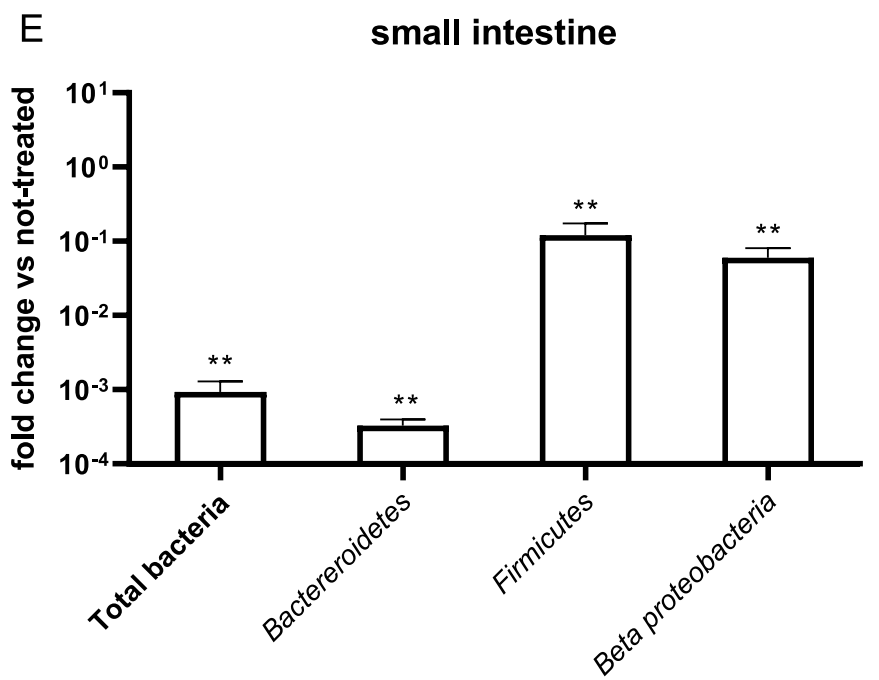

Figure 2. Effect of antibiotic treatment on the bacterial load in mice determined by qPCR on DNA extract of fecal (A-D) pellets and intestinal content (E). Results are shown for Total Bacteria, Bacteroidetes, Firmicutes and Beta proteobacteria as the mean of 5 mice with SEM errors bars. $\left({ }^{* *} \mathrm{p} \leq 0.01\right)$.

are excreted into the intestinal lumen where they are part of the complex mucosal barrier protecting the intestinal epithelial cell layer ${ }^{21}$. AMPs were previously shown to exhibit a toxic effect on $G$. duodenalis trophozoites in vitro ${ }^{22}$. In addition, transcriptional upregulation of $\alpha$-defensins and $\beta$-defensin 1 (defb1) has been observed 21 days following a $G$. muris infection ${ }^{7}$, further indicating their importance. The influence of the microbiome on defensins has been suggested in a previous study as germfree mice displayed strongly reduced expression levels in the small intestine $\mathrm{e}^{23,24}$. Therefore, a qPCR analysis in non-infected control mice was performed to compare expression levels of both total $\alpha$-defensins (defa-tot) and defb 1 between antibiotic treated mice and untreated mice. The results show that for defb1 (Fig. 3C) no effect was visible, while the mRNA expression levels of total $\alpha$-defensins (Fig. 3A) on the other hand displayed a significant downregulation in antibiotic treated mice. After expression, $\alpha$-defensins must be proteolytically cleaved by matrix metalloprotease-7 (MMP-7), as it contains an inhibitory amino-terminal pro-region ${ }^{24}$. It has been shown that mice deficient for MMP-7 show higher G. duodenalis trophozoite counts at day 13 p.i. compared to wild type mice, whilst there was no effect during a G. muris infection ${ }^{25}$. The importance of the intestinal microbiome on the expression levels of MMP-7 has already been studied in the past using germfree mice which showed undetectable levels of MMP-7 by immunohistochemistry ${ }^{26}$. Similarly, in this study we show that antibiotic mediated depletion of the intestinal microbiome results in a significant downregulation of MMP-7 (Fig. 3B). Yet, the biological relevance of this observation remains unclear as Ayabe et al. ${ }^{27}$ showed that basal MMP-7 levels in germ-free mice are already sufficient for the proteolytical cleavage of $\alpha$-defensins. However, this study did not take into account what the effect would be when faced with a potential pathogen.

Other components of the innate immune system that are upregulated following a Giardia infection are the mannose binding lectin 2 (MBL2) and angiogenin 4 (ANG4). MBL2 is a circulating C-type lectin that is able to bind carbohydrate structures on pathogens in order to subsequently activate the complement cascade ${ }^{28}$. Previous studies have shown the importance of MBL2 during a Giardia infection as mice deficient for MBL2 displayed a delayed clearance of both G. duodenalis ${ }^{29}$ and G. muris ${ }^{7}$ compared to wild type mice. However, depletion of the intestinal microbiome actually resulted in an upregulation of MBL2 instead of a downregulation, indicating that the microbiome is not necessary for its expression (Fig. 3D). ANG4 was originally identified as a tumour-derived protein containing angiogenic properties. However, more recently it was also identified as an 
A

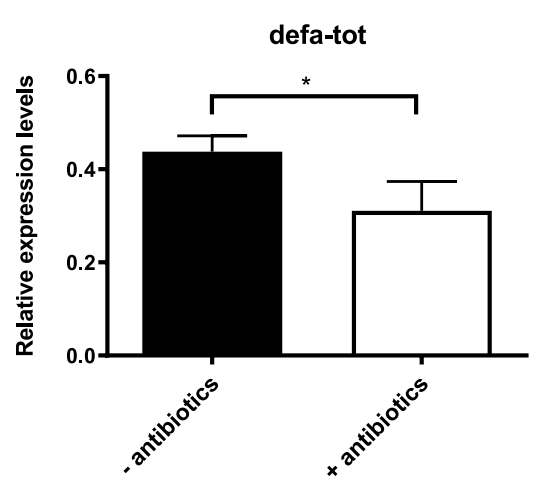

B

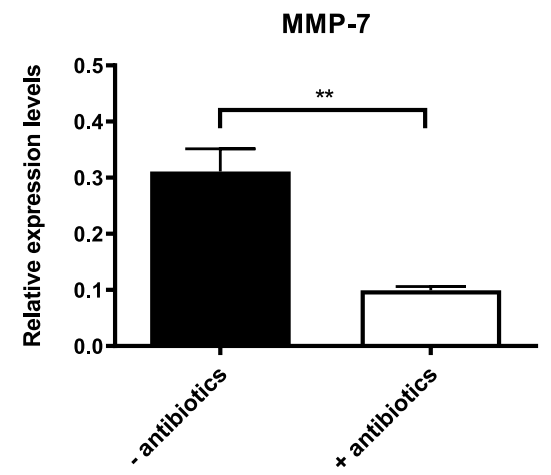

C

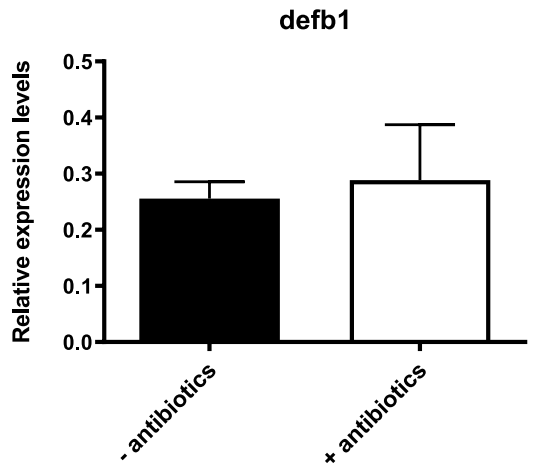

D

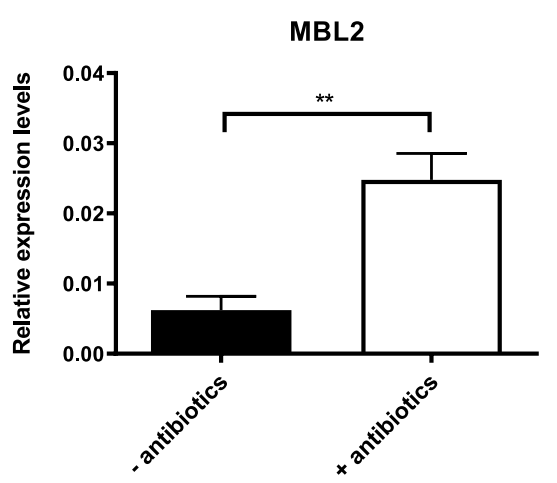

E

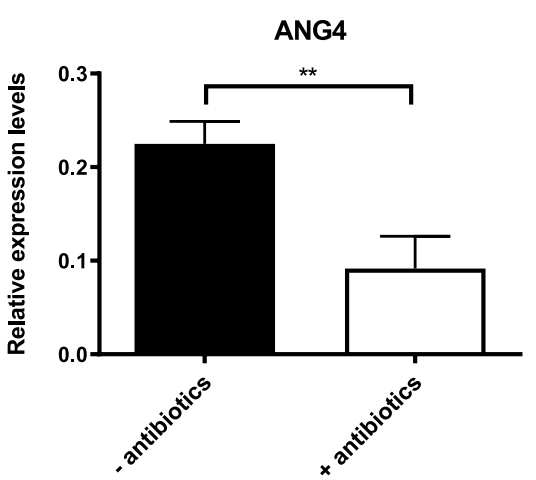

Figure 3. Effect of antibiotic treatment on the transcription of anti-microbial peptides and MBL2 in noninfected control mice without (black bar) and with (white bar) after 5 days of antibiotic cocktail treatment (A) a-defensins (defa-tot), (B) MMP-7, (C) defb1, (D) MBL2 and (E) ANG4. Relative mRNA expression levels were measured by qPCR. Mean transcription level from 5 mice are shown with SEM as error bars. $\left({ }^{\star} \mathrm{p} \leq 0.05\right.$, $\left.{ }^{* *} \mathrm{p} \leq 0.01\right)$.

antimicrobial peptide, produced by Paneth cells, with bactericidal activity against both gram-negative as grampositive bacteria ${ }^{30}$. Interestingly, ANG4 is one of the most upregulated genes following a G. muris infection ${ }^{7}$, yet its role during a Giardia infection still remains unclear. Just like for the other factors of the innate immune response, the expression levels of ANG4 were determined by qPCR between antibiotic treated mice and mice with a conventional microbiome. The results show a significant downregulation of ANG4 due to the absence of an intestinal microbiome resulting in a lower basal level of expression (Fig. 3E). In summary, these results indicate that the presence of several factors of the innate immune response in the intestinal lumen is decreased following antibiotic treatment, which could explain the increased susceptibility of antibiotic treated mice for a G. duodenalis infection.

The intestinal microbiome is not essential for the initiation of the IL-17A response but stimulates IgA production and secretion. In normal conditions, G. muris infected mice develop a protective immune response in which upregulation of IL-17A is necessary for the elimination of the parasite ${ }^{5}$. Antibiotic treated mice however experience difficulties clearing a G. muris infection, suggesting an impaired IL-17 response. To determine the role of the intestinal microbiome on IL-17A induction, the expression levels of both IL-17A and its downstream effector MBL2 were measured by RT-qPCR in both antibiotic-treated and untreated mice following a G. duodenalis or G. muris infection. Previous reports have already shown an upregulation for both IL-17A and MBL2 within the first 3 weeks of a G. muris infection ${ }^{5,7}$. Consistent herewith, Fig. 4A and B demonstrate a significant upregulation of both IL17A and MBL2 at day 21p.i. The upregulation of IL-17A and MBL2 was also observed in the G. muris infected mice treated with the antibiotic cocktail (Fig. 4C,D) and was even stronger, most likely due to the higher parasitic load, in the antibiotic treated mice compared to the untreated mice. These observations indicate that the intestinal microbiome does not influence the induction process itself of the IL-17A response; yet, other downstream effectors of IL-17A in the immune response could still be affected by the microbiome.

One of the most important downstream effectors of IL-17A following a Giardia infection is the stimulation of IgA production by B-cells. The necessity of B-cell development and subsequent IgA production in the protection against Giardia was contested at first, as several studies had contradicting outcomes ${ }^{31,32}$, yet studies performed in IgA deficient mice have shown a strongly delayed clearance of both G. muris as G. duodenalis ${ }^{6}$. To investigate the 
A

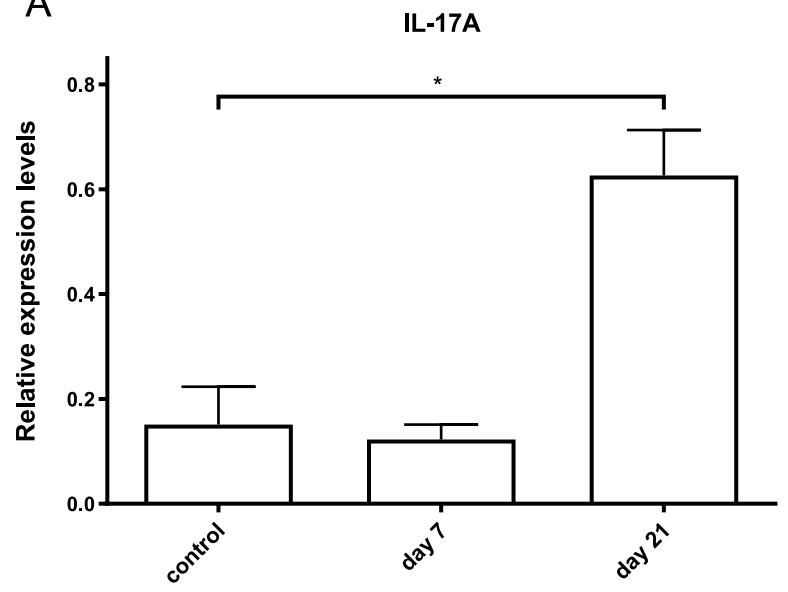

C

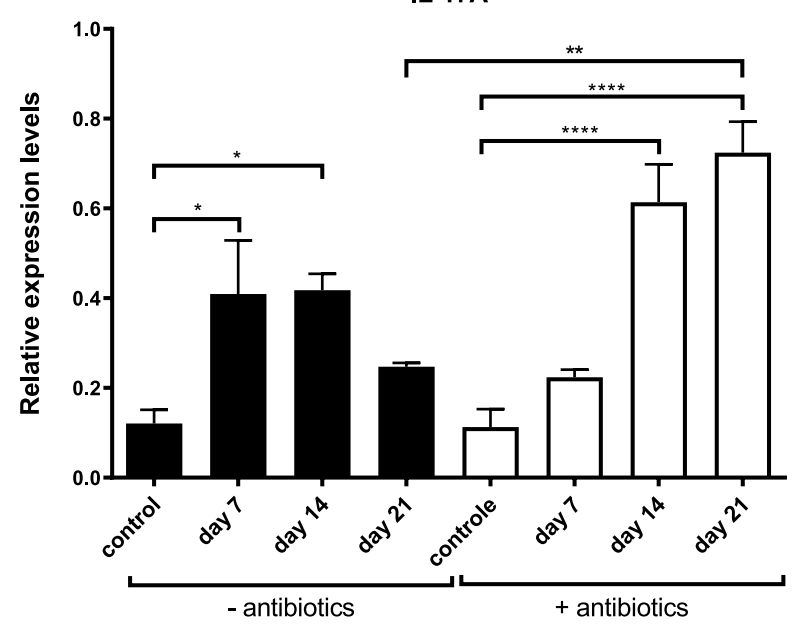

B

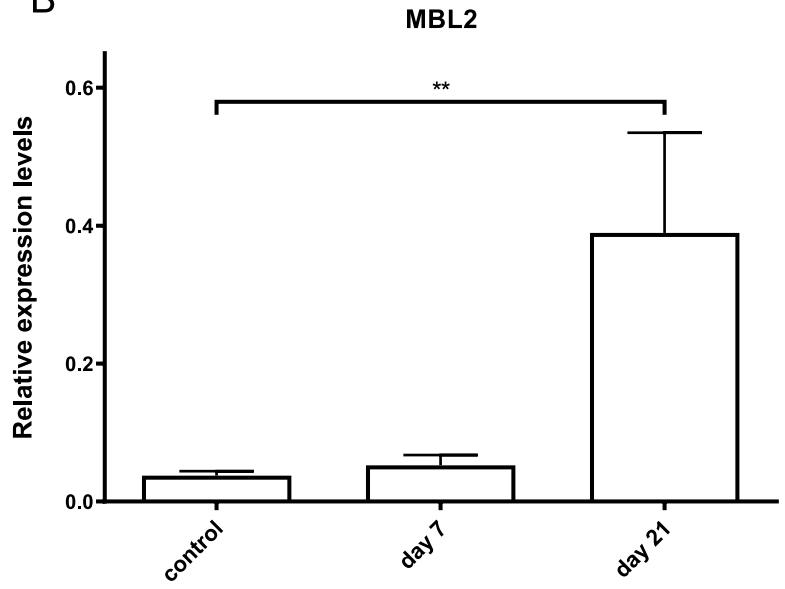

D

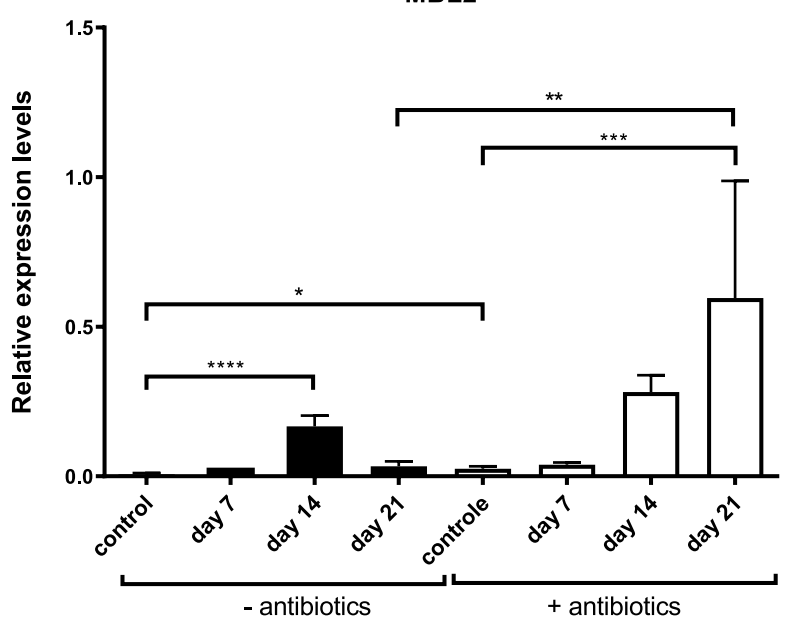

Figure 4. Kinetics of the intestinal IL-17A and MBL2 response following a G. duodenalis (A, B) and G. muris $(\mathbf{C}, \mathbf{D})$ infection in $\mathrm{C} 75 \mathrm{Bl} / 6$ mice without (black bar) and with (white bar) antibiotic cocktail treatment. Relative mRNA expression levels were measured by qPCR at indicated time points. Mean transcription level from 5 mice are shown with SEM as error bars. $\left({ }^{\star} \mathrm{p} \leq 0.05,{ }^{* *} \mathrm{p} \leq 0.01,{ }^{* *} \mathrm{p} \leq 0.001,{ }^{\star * * *} \mathrm{p} \leq 0.0001\right)$.

influence of the microbiome on IgA levels in the intestinal lumen, several approaches were used in this study. In a first step, flow cytometry was performed to analyse the distribution of several B-cell types in the Peyer's patches of the small intestine. Figure 5B shows that microbiome depletion did not result in altered amounts of total B-cells, yet, the amount of IgA-producing B-cells was significantly decreased in the antibiotic treated mice, both in control and infected mice (Fig. 5A). Besides IgA-expressing B-cells, also IgM positive B-cells were decreased due to the antibiotic treatment but only between the control groups (Supplementary Data S3), indicating a compensation of other B-cell subpopulations for the decrease in IgA ${ }^{+}$and $\operatorname{IgM}^{+}$while total B-cells remained equal. Second, the expression levels of the polymeric immunoglobulin A receptor (PIgR) were examined by qRT-PCR. PIgR is an essential transporter for IgA in order to translocate from the lamina propria, where it is produced by B-cells, to the intestinal lumen ${ }^{33}$. Previous studies have shown that also PIgR is partially regulated by an IL-17 induction ${ }^{34}$. As observed in Fig. 5C, intestinal microbiome depletion led to a significant downregulation of PIgR in control mice at day 14 p.i. and hinted to a downward trend in infected mice. Collectively, the above findings suggest that antibiotic-mediated depletion of the microbiome causes a decrease in luminal $\operatorname{IgA}$ as a result of decreased numbers of Ig $\mathrm{A}^{+} \mathrm{B}$-cells in the Peyer's patches concomitant with IgA transporter downregulation. In order to confirm this hypothesis, total IgA levels in the intestinal lumen were measured by an IgA ELISA on luminal extracts isolated at day 21 p.i. (Fig. 5D). As described in previous studies, the amount of luminal IgA increases in the mice both with and without antibiotics when infected with G. muris. However, intestinal microbiome depletion results in lower IgA levels in control as well as in infected mice, which could explain the reduced clearance of a G. muris infection following antibiotic treatment.

Gut microbiota influences the intestinal motility. An often forgotten part of the intestinal immune response is the expulsion of invading pathogens by the infected host through increased intestinal motility. This process is a complex regulated system depending on multiple factors. Studies in the past have shown that several 
A

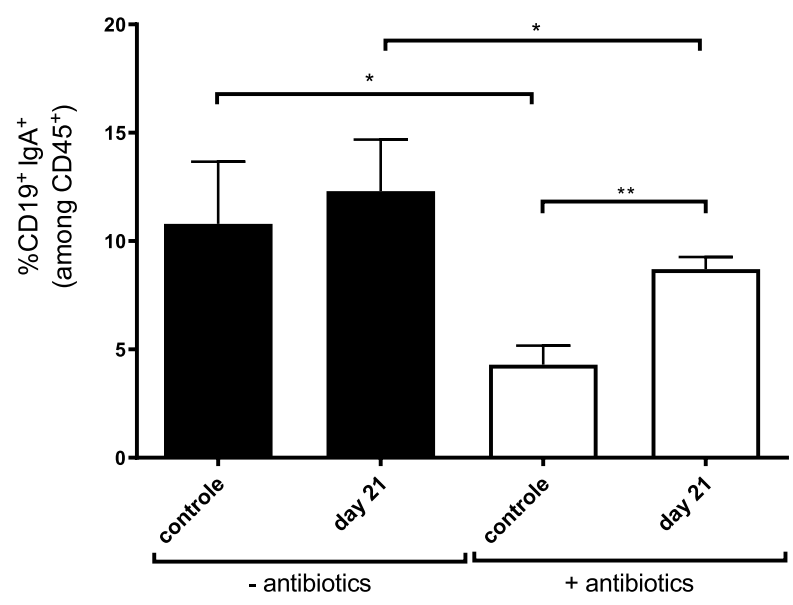

C

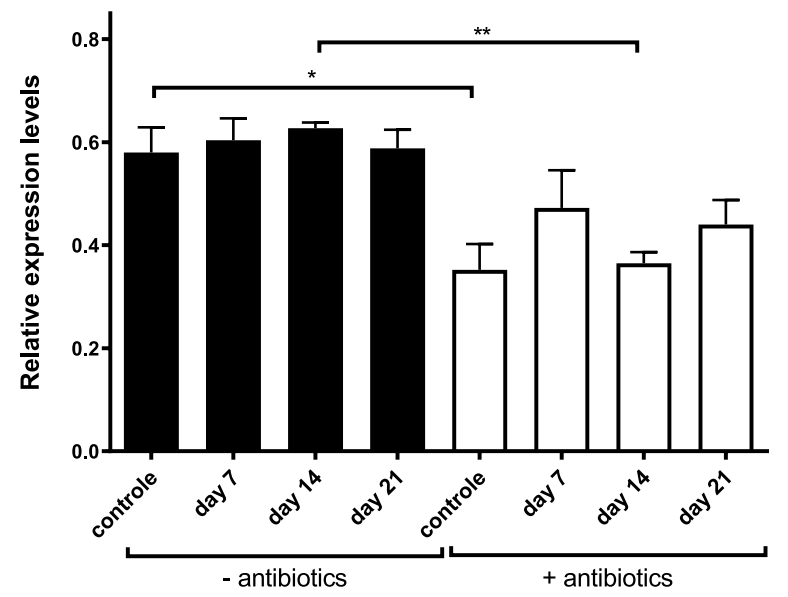

B

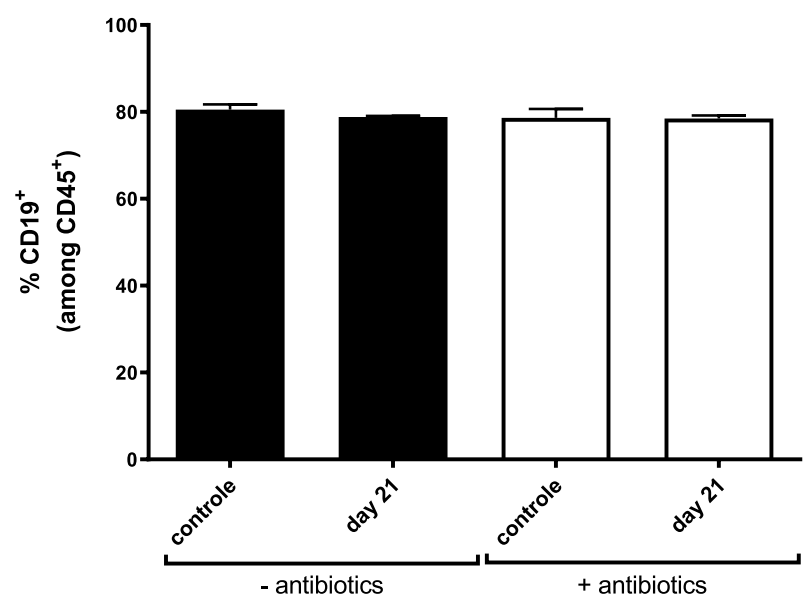

$\mathrm{D}$

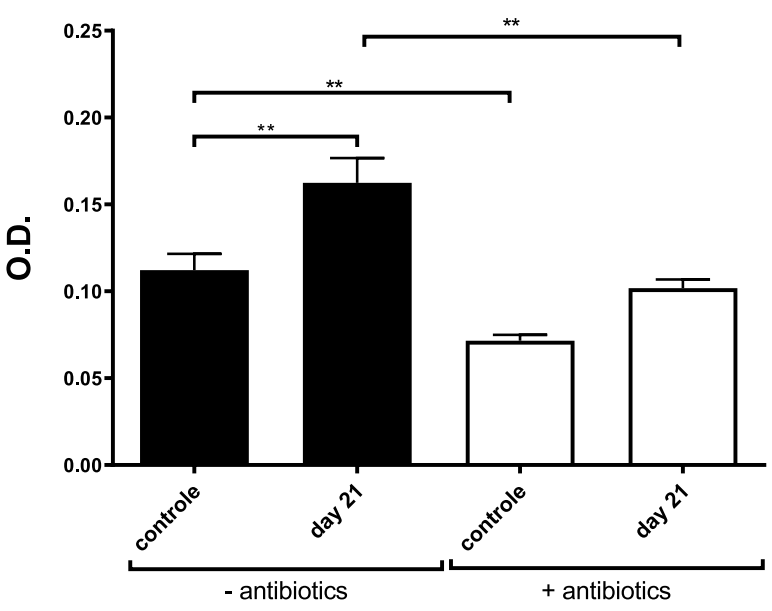

Figure 5. Effect of antibiotic treatment and G. muris infection on the intestinal production and secretion of IgA. (A) Relative percentage of IgA producing B-cells within the $\mathrm{CD}^{2} 5^{+}$cell population isolated from the Peyer's patch tissue. (B) Relative percentage of total B-cells within the $\mathrm{CD}^{4} 5^{+}$cell population isolated from the Peyer's patch tissue. (C) Relative mRNA expression levels of PIgR measured by qPCR at indicated time points. (D) Total intestinal IgA levels. Results are shown as the mean from 5 mice with SEM as error bars. $\left({ }^{\star} \mathrm{p} \leq 0.05,{ }^{* *} \mathrm{p} \leq 0.01\right)$.

intestinal pathogenic infections, including Giardia, led to an increased intestinal motility, in which the host attempts to clear the infection rapidly ${ }^{35,36}$.

As stated above, gut microbiome depletion induces severe physiological changes of the digestive tract. These findings led to the consideration that depletion possibly alters the intestinal motility as well. To test this hypothesis, intestinal motility was determined by the distance an orally administrated dye travelled through the digestive tract following infection with G. muris (Fig. 6A). Unlike previous studies, our results do not show increased intestinal motility upon a G. muris infection. Yet, gut microbiome depletion by an antibiotic treatment did impact intestinal motility. Both in the non-infected controls as in the G. muris infected animals, treatment led a longer intestinal transit time (Fig. 6B). As a consequence of decreased intestinal motility, Giardia trophozoites are more slowly expulsed from the small intestine and subsequently may reside longer in the intestinal lumen. Together with a compromised immune response (as indicated by the results described above), these findings may allow explaining the prolonged phenotype of a G. muris infection and the increased susceptibility of antibiotic-treated mice to a G. duodenalis infection.

\section{Conclusion}

The outcome of this study clearly highlights the importance of the intestinal microbiome in the Giardia-mice immune interaction. Not only did the G. muris infection in microbiome-depleted mice show a more chronic course over time, it also resulted in a higher parasite burden. Surprisingly, the IL-17A induction mediated by Giardia was still intact suggesting that IL-17A upregulation occurs independently of an intact intestinal microbiome. Nonetheless, several immune effectors mechanisms were compromised in the absence of the intestinal microbiota. Those factors belonged to both innate (AMPs and intestinal transit) and adaptive (IgA) immune 
A

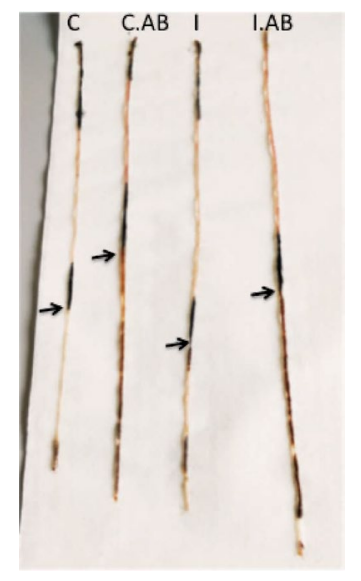

B

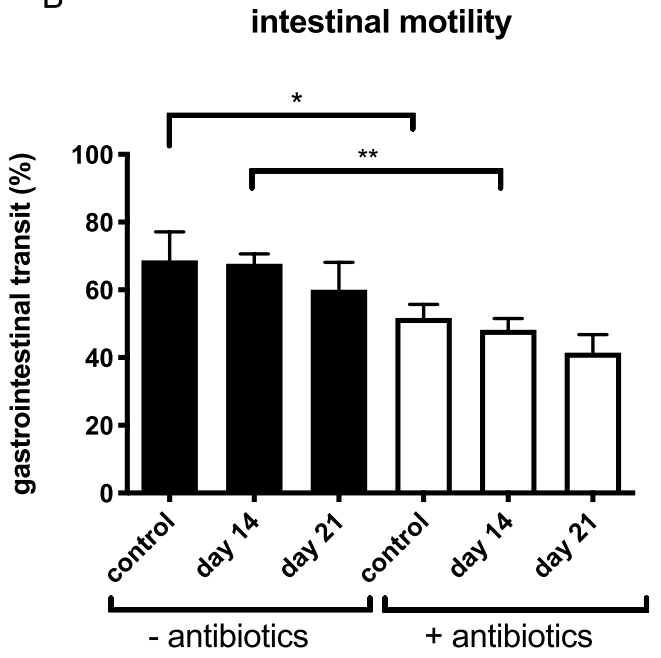

Figure 6. Effect of antibiotic treatment and G. muris infection on the intestinal motility. (A) Picture of murine small intestine, 20 min after oral administration of $10 \%$ charcoal solution. (B) Kinetics of intestinal transit following a G. muris infection in C75 BL/6 mice without (black bar) and with (white bar) antibiotic treatment. Results are shown as the mean from 5 mice with SEM as error bars. $\left({ }^{*} \mathrm{p} \leq 0.05,{ }^{* *} \mathrm{p} \leq 0.01\right)$.

responses. Our findings show that IL-17A induction on its own is not sufficient for clearance of a G. muris infection but additionally requires priming of the immune system by the intestinal microbiome. Furthermore, the decrease of components important in the innate immune system, such as the defensins, Ang 4 and intestinal motility, could potentially explain the increased susceptibility of microbiome-depleted mice to a G. duodenalis infection.

In addition to the new insights into the interaction between the parasite, the microbiome and the host immune system, the outcome of this study also highlights the potential danger of antibiotic usage during a Giardia infection. Giardiasis is often misdiagnosed at first, resulting in the prescription of antibiotics which are ineffective against Giardia ${ }^{37}$. Even apart from the issues concerning antibiotic resistance, the results presented here show that mistreatment of Giardia with antibiotics could result in a more severe and prolonged infection. Importantly however, the duration of such an effect, after the termination of the antibiotic treatment, is currently not known and requires further research.

Received: 12 August 2020; Accepted: 10 May 2021

Published online: 19 May 2021

\section{References}

1. Flanagan, P. Giardia-diagnosis, clinical course and epidemiology: A review. Epidemiol. Infect/ 109, 1 (1992).

2. Farthing, M. J. Giardiasis. Gastroenterol. Clin. 25, 493-515 (1996).

3. Reh, L. et al. Substantial prevalence of enteroparasites Cryptosporidium spp., Giardia duodenalis and Blastocystis sp. in asymptomatic schoolchildren in Madrid, Spain, November 2017 to June 2018. Eurosurveillance 24, 43 (2019).

4. Dann, S. M. et al. IL-17A promotes protective IgA responses and expression of other potential effectors against the lumen-dwelling enteric parasite Giardia. Exp. Parasitol. 156, 68-78 (2015).

5. Dreesen, L. et al. Giardia muris infection in mice is associated with a protective interleukin 17A response and induction of peroxisome proliferator-activated receptor alpha. Infect. Immun. 82, 3333-3340 (2014).

6. Langford, T. D. et al. Central importance of immunoglobulin A in host defense against Giardia spp... Infect. Immun. 70, 11-18 (2002).

7. Paerewijck, O. et al. Interleukin-17 receptor A (IL-17RA) as a central regulator of the protective immune response against Giardia. Sci. Rep. 7, 1-14 (2017).

8. Manel, N., Unutmaz, D. \& Littman, D. R. The differentiation of human T H-17 cells requires transforming growth factor- $\beta$ and induction of the nuclear receptor ROR $\gamma$ t. Nat. Immunol. 9, 641 (2008).

9. Ivanov, I. I. et al. The orphan nuclear receptor ROR $\gamma$ t directs the differentiation program of proinflammatory IL-17+ T helper cells. Cell 126, 1121-1133 (2006).

10. Ivanov, I. I. et al. Specific microbiota direct the differentiation of IL-17-producing T-helper cells in the mucosa of the small intestine. Cell Host Microbe 4, 337-349 (2008).

11. Dzidic, M., Boix-Amorós, A., Selma-Royo, M., Mira, A. \& Collado, M. C. Gut microbiota and mucosal immunity in the neonate. Med. Sci. 6, 56 (2018).

12. Gensollen, T., Iyer, S. S., Kasper, D. L. \& Blumberg, R. S. How colonization by microbiota in early life shapes the immune system. Science 352, 539-544 (2016).

13. Paerewijck, O., Maertens, B., Gagnaire, A., De Bosscher, K. \& Geldhof, P. Delayed development of the protective IL-17A response following a Giardia muris infection in neonatal mice. Sci. Rep. 9, 1-7 (2019).

14. Singer, S. M. \& Nash, T. E. The role of normal flora in Giardia lamblia infections in mice. J. Infect. Dis. 181, 1510-1512 (2000).

15. Roberts-Thomson, I. C. \& Mitchell, G. F. Giardiasis in mice: I. Prolonged infections in certain mouse strains and hypothymic (nude) mice. Gastroenterology 75, 42-46 (1978). 
16. Bonnardel, J. et al. Innate and adaptive immune functions of peyer's patch monocyte-derived cells. Cell Rep. 11, 770-784 (2015).

17. Belosevic, M., Faubert, G., MacLean, J., Law, C. \& Croll, N. Giardia lamblia infections in Mongolian gerbils: an animal model. J. Infect. Dis. 147, 222-226 (1983).

18. Byrd, L. G., Conrad, J. T. \& Nash, T. E. Giardia lamblia infections in adult mice. Infect. Immun. 62, 3583-3585 (1994).

19. Reikvam, D. H. et al. Depletion of murine intestinal microbiota: effects on gut mucosa and epithelial gene expression. PLoS ONE 6, e17669 (2011)

20. Round, J. L. \& Mazmanian, S. K. The gut microbiota shapes intestinal immune responses during health and disease. Nat. Rev. Immunol. 9, 313-323 (2009).

21. Bevins, C. L. \& Salzman, N. H. Paneth cells, antimicrobial peptides and maintenance of intestinal homeostasis. Nat. Rev. Microbiol. 9, 356-368 (2011).

22. Aley, S. B., Zimmerman, M., Hetsko, M., Selsted, M. E. \& Gillin, F. D. Killing of Giardia lamblia by cryptdins and cationic neutrophil peptides. Infect. Immun. 62, 5397-5403 (1994).

23. Sugi, Y. et al. $\alpha$-Defensin 5 gene expression is regulated by gut microbial metabolites. Biosci. Biotechnol. Biochem. 81, 242-248 (2017).

24. Wilson, C. L. et al. Differential processing of $\alpha$-and $\beta$-defensin precursors by matrix metalloproteinase-7 (MMP-7). J. Biol. Chem. 284, 8301-8311 (2009).

25. Eckmann, L. Mucosal defences against Giardia. Parasite Immunol. 25, 259-270 (2003).

26. López-Boado, Y. S. et al. Bacterial exposure induces and activates matrilysin in mucosal epithelial cells. J. Cell Biol. 148, 1305-1315 (2000).

27. Ayabe, T. et al. Activation of Paneth cell a-defensins in mouse small intestine. J. Biol. Chem. 277, 5219-5228 (2002).

28. Eddie Ip, W., Takahashi, K., Alan Ezekowitz, R. \& Stuart, L. M. Mannose-binding lectin and innate immunity. Immunol. Rev. 230, 9-21 (2009).

29. Tako, E. A., Hassimi, M. F., Li, E. \& Singer, S. M. Transcriptomic analysis of the host response to Giardia duodenalis infection reveals redundant mechanisms for parasite control. MBio 4, e00660-e60013 (2013).

30. Hooper, L. V., Stappenbeck, T. S., Hong, C. V. \& Gordon, J. I. Angiogenins: A new class of microbicidal proteins involved in innate immunity. Nat. Immunol. 4, 269-273 (2003).

31. Singer, S. M. \& Nash, T. E. T-cell-dependent control of acute Giardia lamblia infections in mice. Infect. Immun. 68, 170-175 (2000).

32. Stager, S. \& Muller, N. Giardia lamblia infections in B-cell-deficient transgenic mice. Infect. Immun. 65, 3944-3946 (1997).

33. Turula, H. \& Wobus, C. E. The role of the polymeric immunoglobulin receptor and secretory immunoglobulins during mucosal infection and immunity. Viruses 10, 237 (2018).

34. Cao, A. T., Yao, S., Gong, B., Elson, C. O. \& Cong, Y. Th17 cells upregulate polymeric Ig receptor and intestinal IgA and contribute to intestinal homeostasis. J. Immunol. 189, 4666-4673 (2012).

35. Li, E., Zhou, P. \& Singer, S. M. Neuronal nitric oxide synthase is necessary for elimination of Giardia lamblia infections in mice. J. Immunol. 176, 516-521 (2006).

36. Vallance, B. A., Blennerhassett, P. A., Huizinga, J. D. \& Collins, S. M. Mast cell-independent impairment of host defense and muscle contraction in T. spiralis-infected W/WVmice. Am. J. Physiol. 280, 640-648 (2001).

37. Beer, K. D., Collier, S. A., Du, F. \& Gargano, J. W. Giardiasis diagnosis and treatment practices among commercially insured persons in the United States. Clin. Infect. Dis. 64, 1244-1250 (2017).

\section{Author contributions}

Conception or design of the work: B.M., A.G., O.P., P.G. Data collection: B.M., A.G., O.P. Data analysis and interpretation: B.M., A.G., K.D.B., P.G. Drafting the article: B.M, A.G., P.G. Critical revision of the article: B.M., A.G., K.D.B., P.G. Final approval of the version to be published: all authors.

\section{Funding}

Funding was provided by Fonds Wetenschappelijk Onderzoek and Bijzonder Onderzoeksfonds UGent.

\section{Competing interests}

The authors declare no competing interests.

\section{Additional information}

Supplementary Information The online version contains supplementary material available at https://doi.org/ 10.1038/s41598-021-90261-Z.

Correspondence and requests for materials should be addressed to P.G.

Reprints and permissions information is available at www.nature.com/reprints.

Publisher's note Springer Nature remains neutral with regard to jurisdictional claims in published maps and institutional affiliations.

Open Access This article is licensed under a Creative Commons Attribution 4.0 International License, which permits use, sharing, adaptation, distribution and reproduction in any medium or format, as long as you give appropriate credit to the original author(s) and the source, provide a link to the Creative Commons licence, and indicate if changes were made. The images or other third party material in this article are included in the article's Creative Commons licence, unless indicated otherwise in a credit line to the material. If material is not included in the article's Creative Commons licence and your intended use is not permitted by statutory regulation or exceeds the permitted use, you will need to obtain permission directly from the copyright holder. To view a copy of this licence, visit http://creativecommons.org/licenses/by/4.0/.

(c) The Author(s) 2021 